\title{
Temporal Gliosarcoma With Extraneural Metastasis -Case Report-
}

\author{
Daisuke MAEDA, Takahito MIYAZAWA, \\ Terushige TOYOOKA, and Katsuji SHIMA \\ Department of Neurosurgery, National Defense Medical College, Tokorozawa, Saitama
}

\begin{abstract}
A 51-year-old woman presented with a rare case of temporal gliosarcoma manifesting as a 2-month history of headache that rapidly penetrated the middle fossa floor postoperatively and metastasized to the lung. The tumor included an anteroinferior component consisting of a sarcomatous lesion adjacent to the middle fossa floor, and a posterosuperior component consisting of a gliomatous lesion. The MIB-1 index of the sarcomatous component was $47.5 \%$, and that of the gliomatous component was $36.5 \%$. In addition to the highly proliferative nature of the sarcomatous component, the craniotomy with partial excision of the dura mater might have accelerated the tumor penetration through the temporal base and the hematogenous metastasis to the lung.
\end{abstract}

Key words: temporal gliosarcoma, extraneural metastasis, MIB-1 index

\section{Introduction}

Gliosarcoma is a rare brain tumor with both gliomatous and sarcomatous components. Recent molecular genetic studies have suggested that these components originate from monoclonal precursor cells. ${ }^{2)}$ Dural invasion and extracranial metastasis are more common in gliosarcoma than glioblastoma. ${ }^{9}$ Here, we report a rare case of gliosarcoma that originated in the temporal lobe, and postoperatively extended into the infratemporal fossa through the middle fossa floor at recurrence, and finally metastasized to the lung.

\section{Case Report}

A 51-year-old woman was admitted to our hospital with a 2-month history of headache. Magnetic resonance (MR) imaging revealed an irregular enhanced mass in the right temporal lobe with two components: the anteroinferior part consisted of a 4-cm solid mass with heterogeneous enhancement adjacent to the middle fossa floor, and the posterosuperior part consisted of a 3-cm cystic mass with ring-like enhancement. The dura mater attached to the anteroinferior part showed enhancement, but no dural tail sign or extracranial extension was seen (Fig. 1).

Gross total removal of the tumor was performed using temporal lobectomy. The anterior part of the tumor was tightly adhered to the dura mater of the middle fossa. A small piece of the invaded dura mater was removed and patched using temporal fascia. No infratemporal tumor growth was identified during surgery. The dura mater was

Received April 6, 2009; Accepted August 10, 2009
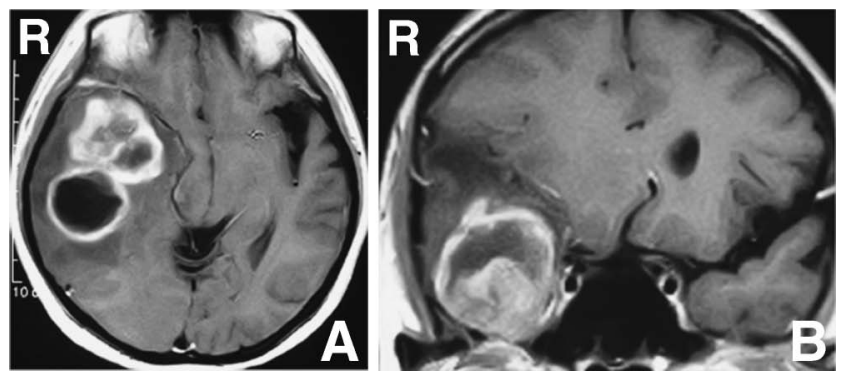

Fig. 1 Axial (A) and coronal (B) $T_{1}$-weighted magnetic resonance images with gadolinium revealing a heterogeneously enhanced lesion in the right temporal lobe. The lesion is attached to the middle fossa floor with contrast enhancement of the adjacent dura mater, but no dural tail sign or extracranial extension is seen. The anteroinferior part consists of a $4-\mathrm{cm}$ solid mass with irregular enhancement adjacent to the middle fossa floor and the posterosuperior part consists of a $3-\mathrm{cm}$ cystic mass with ring-like enhancement.

demarcated from the attached tumor and so was not radically excised.

The histological diagnosis was gliosarcoma based on a biphasic pattern consisting of gliomatous and sarcomatous components. The postero-superior part of the tumor was primarily gliomatous, and the antero-inferior part included atypical mesenchymal spindle cell proliferation (Fig. 2B, D). The tumor attached to the middle fossa floor was exclusively sarcomatous. Ki-67 immunohistochemistry showed the MIB-1 scores were $47.5 \%$ for the sarcomatous component and $36.5 \%$ for the gliomatous component (Fig. 2E, F). 


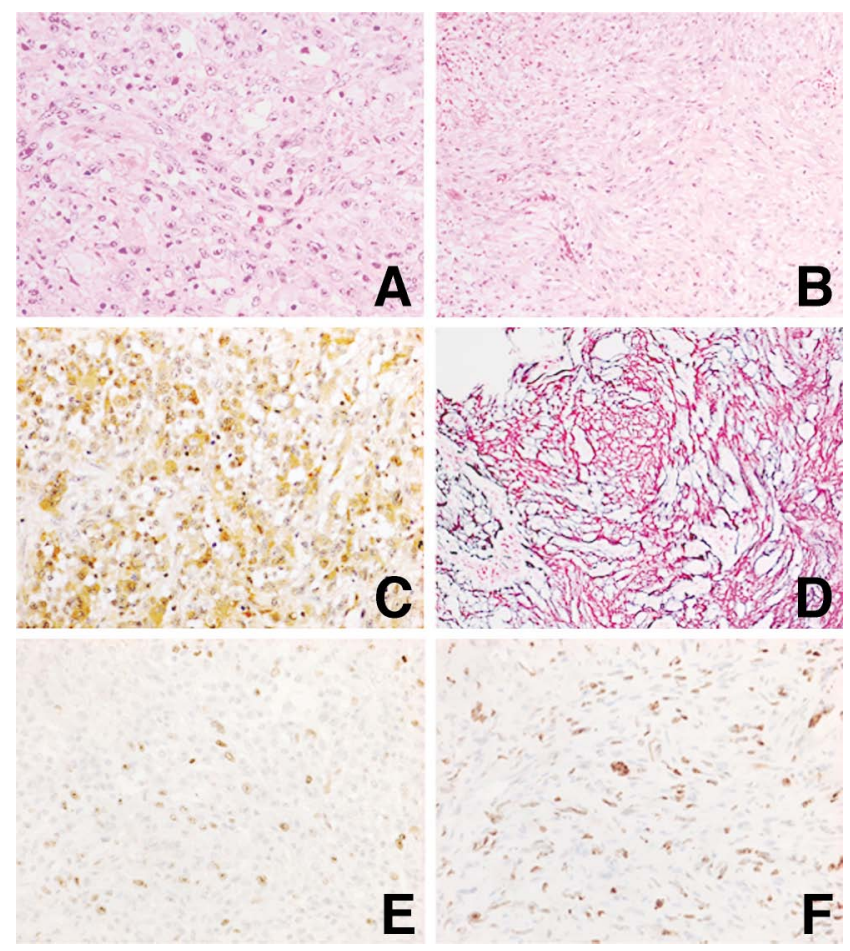

Fig. 2 Photomicrographs showing the typical gliomatous (A, $\mathrm{C}, \mathrm{E})$ and sarcomatous (B, D, F) components of the gliosarcoma. A: Typical findings of malignant glioma, with diffuse proliferation of pleomorphic cells varying in size with nuclear atypia, hyperchromasia or necrotic cells with chromatin condensation. B: Atypical mesenchymal spindle cell proliferation, with a pleomorphic nuclei formation of whorled fascicles and a herringbone pattern. C: Large number of glial fibrillary acidic protein (GFAP)-immunopositive cells. D: Abundant sarcomatous reticulin fibers. E, F: MIB-1 index calculated as the ratio of Ki-67-immunopositive cells was $36.5 \%$ for the gliomatous component (E) and $\mathbf{4 7 . 5 \%}$ for the sarcomatous component ( $F$ ). Hematoxylin-eosin stain (A, B), GFAP immunoreactive stain (C), reticulin stain (D), and Ki-67 immunoreactive stain (E, F), original magnification $\times 400(\mathrm{~A}, \mathrm{C}, \mathrm{E}, \mathrm{F})$ and $\times 200(\mathrm{~B}, \mathrm{D})$.

The patient underwent chemotherapy with intravenous administration of ACNU and local irradiation (60 Gy) to the right temporal region. Follow-up MR imaging revealed a recurrent lesion in the intracranial temporal base after 2 months. Despite additional chemotherapy and irradiation, the tumor grew rapidly, penetrated the middle fossa base, and extended into the infratemporal fossa (Fig. 3). Furthermore, 4 months after admission, chest computed tomography showed multiple nodular lesions in the right lung indicating metastasis (Fig. 4). She died of respiratory failure 5 months after the initial diagnosis.

\section{Discussion}

Clinical outcome does not significantly differ between gliosarcoma and glioblastoma, ${ }^{5,10)}$ possibly because the gliomatous and sarcomatous components of gliosarcoma have a genetically common origin. Patients with highly sarcomatous gliosarcomas have recently survived longer
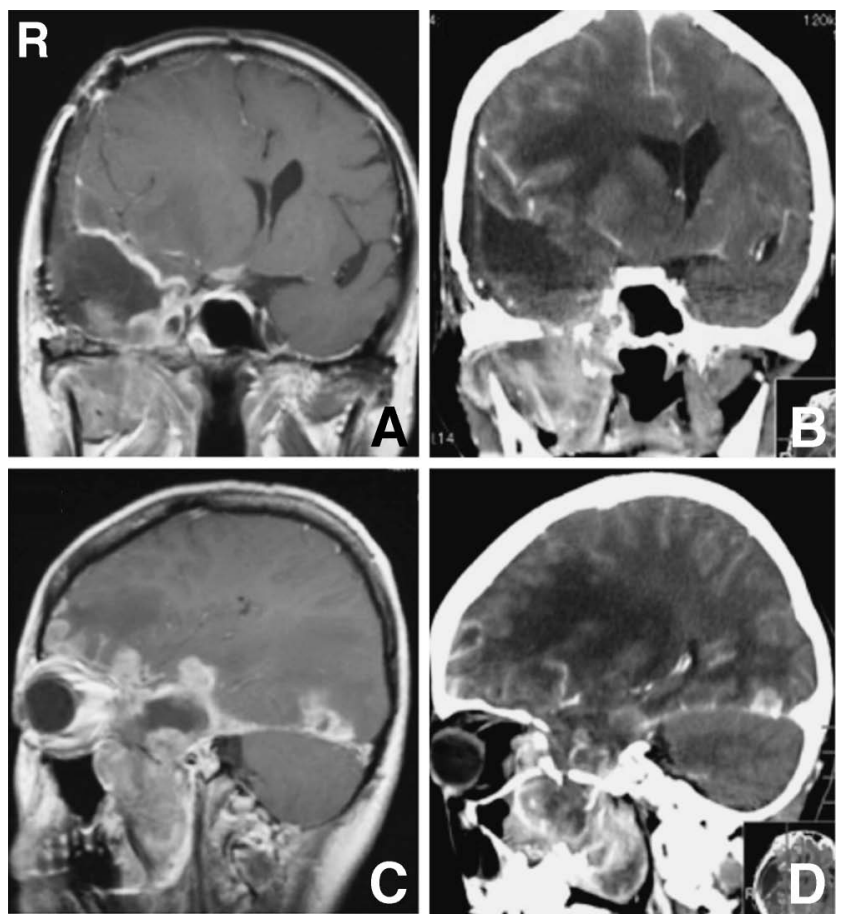

Fig. 3 Coronal (A) and sagittal (C) $T_{1}$-weighted magnetic resonance images with gadolinium obtained 2 months after the operation revealing recurrent infratemporal lesions adjacent to the middle fossa floor, and extending into the infratemporal fossa. Coronal (B) and sagittal (D) computed tomography scans revealing that the bony structures of the middle fossa floor were destroyed by the tumor.

than patients with predominantly gliomatous gliosarcomas. ${ }^{4,11)}$ In the present case, the sarcomatous component was predominant but the tumor showed rapid recurrence, with extension into the infratemporal fossa and lung metastasis. Both the disease-free survival time and the actual survival time were extremely short.

Grading systems using the MIB-1 score are strongly prognostic in patients with soft tissue sarcoma of the extremities and trunk. ${ }^{6)}$ The MIB-1 index of gliosarcomas ranged from $7.7 \%$ to $36.1 \%{ }^{7)}$ and was a prognostic factor for glioblastoma. ${ }^{8)}$ In our case, the MIB-1 score of the sarcomatous component $(47.5 \%)$ was higher than that of the gliomatous component (36.5\%). The most proliferative part of the tumor was present in the sarcomatous component attached to the dura mater of the middle fossa base. The MIB-1 index of this part was 50\%. This unusual feature and the severe prognosis were probably caused by the highly proliferative nature of the tumor cells based on the MIB-1 index, particularly in the sarcomatous component adjacent to the temporal base, in addition to the initial infiltrative nature of this tumor.

Extraneural metastases have been described in $11 \%$ of previously reported cases of gliosarcoma, most commonly involving the lungs (72\%), liver (41\%), and lymph nodes $(18 \%)^{1,9)}$ Gliosarcoma tends to disseminate hematogenous metastases more frequently than glioblastoma due to the mesenchymal component. ${ }^{3)}$ Hematogenous metastases 


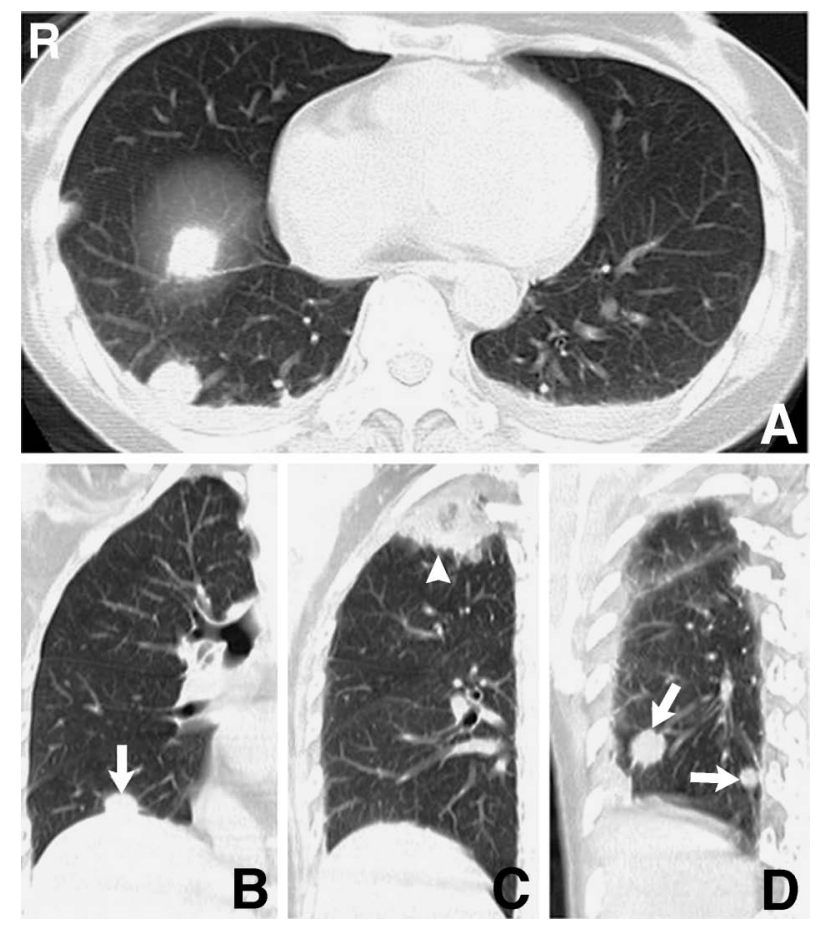

Fig. 4 Axial (A) and coronal (B-D) chest computed tomography scans at the lung window at 4 months after the initial diagnosis of gliosarcoma. A: Two nodular masses in the right lung with bilateral pleural effusions. B-D: Multiple coin lesions (arrows) in the right lung with atelectasis of the upper lobe (arrowhead).

may result from invasion of the intratumoral vessels and transdural penetration into the dural sinuses caused by craniotomy procedure or cranial irradiation. ${ }^{9)}$ Cranial irradiation prolongs survival in patients with glioblastoma, but may also facilitate sarcomatous metaplasia with acquisition of an angioinvasive phenotype..$^{1,3)}$

In our case, craniotomy with partial excision of the dura matter and local irradiation may have accelerated tumor penetration through the temporal base and hematogenous metastasis to the lung. The present case suggests that surgeons who identify dural tumor invasion in the temporal base should excise the attached dura as radically as possible with repair of the cranial base to prevent postoperative infratemporal tumor extension and extraneural metastasis.

\section{References}

1) Beaumont TL, Kupsky WJ, Barger GR, Sloan AE: Gliosarcoma with multiple extracranial metastases: case report and review of the literature. J Neurooncol 83: 39-46, 2007

2) Boerman RH, Anderl K, Herath J, Borell T, Johnson N, Schaeffer-Klein J, Kirchhof A, Raap AK, Scheithauer BW, Jenkins RB: The glial and mesenchymal elements of gliosarcomas share similar genetic alterations. J Neuropathol Exp Neurol 55: 973-981, 1996

3) Cerame MA, Guthikonda M, Kohli CM: Extraneural metastases in gliosarcoma: a case report and review of the literature. Neurosurgery 17: 413-418, 1985

4) Cervoni L, Celli P: Cerebral gliosarcoma: prognostic factors. Neurosurg Rev 19: 93-96, 1996

5) Galanis E, Buckner JC, Dinapoli RP, Scheithauer BW, Jenkins RB, Wang CH, O'Fallon JR, Farr G Jr: Clinical outcome of gliosarcoma compared with glioblastoma multiforme: North Central Cancer Treatment Group results. J Neurosurg 89: 425-430, 1998

6) Hasegawa T, Yamamoto S, Yokoyama R, Umeda T, Matsuno Y, Hirohashi S: Prognostic significance of grading and staging systems using MIB-1 score in adult patients with soft tissue sarcoma of the extremities and trunk. Cancer 95: 843-851, 2002

7) Horiguchi H, Hirose T, Kannuki S, Nagahiro S, Sano T: Gliosarcoma: an immunohistochemical, ultrastructural and fluorescence in situ hybridization study. Pathol Int 48: 595-602, 1998

8) Kleinschmidt-DeMasters BK, Meltesen L, McGavran L, Lillehei KO: Characterization of glioblastomas in young adults. Brain Pathol 16: 273-286, 2006

9) Matsuyama J, Mori T, Hori S, Nakano T, Yamada A: Gliosarcoma with multiple extracranial metastases. Case report. Neurol Med Chir (Tokyo) 29: 938-943, 1989

10) Meis JM, Martz KL, Nelson JS: Mixed glioblastoma multiforme and sarcoma. A clinicopathologic study of 26 radiation therapy oncology group cases. Cancer 67: 2342-2349, 1991

11) Salvati M, Caroli E, Raco A, Giangaspero F, Delfini R, Ferrante L: Gliosarcomas: analysis of 11 cases do two subtypes exist? J Neurooncol 74: 59-63, 2005

Address reprint requests to: Terushige Toyooka, M.D., Department of Neurosurgery, National Defense Medical College, 3-2 Namiki, Tokorozawa, Saitama 359-8513, Japan. e-mail: teru_toy1809@friend.ocn.ne.jp 